\title{
Video Article \\ High-Contrast and Fast Photorheological Switching of a Twist-Bend Nematic Liquid Crystal
}

\author{
Satoshi Aya ${ }^{1}$, Péter Salamon ${ }^{2}$, Daniel A. Paterson ${ }^{3}$, John M. D. Storey ${ }^{3}$, Corrie T. Imrie ${ }^{3}$, Fumito Araoka ${ }^{1}$, Antal Jákli ${ }^{4}$ Ágnes Buka ${ }^{2}$ \\ ${ }^{1}$ RIKEN Center for Emergent Matter Science (CEMS) \\ ${ }^{2}$ Institute for Solid State Physics and Optics, Wigner Research Center for Physics, Hungarian Academy of Sciences \\ ${ }^{3}$ Department of Chemistry, School of Natural and Computing Sciences, University of Aberdeen \\ ${ }^{4}$ Chemical Physics Interdisciplinary Program and Liquid Crystal Institute, Kent State University
}

Correspondence to: Satoshi Aya at satoshiaya@scut.edu.cn, Fumito Araoka at fumito.araoka@riken.jp

URL: https://www.jove.com/video/60433

DOI: doi: $10.3791 / 60433$

Keywords: Chemistry, Issue 152, liquid crystal, twist-bend nematic phase, azobenzene, photo-rheology, solidification-liquefaction switching, polarized light microscopy, photo-differential scanning calorimetry, X-ray diffraction

Date Published: 10/31/2019

Citation: Aya, S., Salamon, P., Paterson, D.A., Storey, J.M., Imrie, C.T., Araoka, F., Jákli, A., Buka, Á. High-Contrast and Fast Photorheological Switching of a Twist-Bend Nematic Liquid Crystal. J. Vis. Exp. (152), e60433, doi:10.3791/60433 (2019).

\section{Abstract}

Smart viscoelastic materials that respond to specific stimuli are one of the most attractive classes of materials important to future technologies, such as on-demand switchable adhesion technologies, actuators, molecular clutches, and nano-/microscopic mass transporters. Recently it was found that through a special solid-liquid transition, rheological properties can exhibit significant changes, thus providing suitable smart viscoelastic materials. However, designing materials with such a property is complex, and forward and backward switching times are usually long. Therefore, it is important to explore new working mechanisms to realize solid-liquid transitions, shorten the switching time, and enhance the contrast of rheological properties during switching. Here, a light-induced crystal-liquid phase transition is observed, which is characterized by means of polarizing light microscopy (POM), photorheometry, photo-differential scanning calorimetry (photo-DSC), and X-ray diffraction (XRD). The light-induced crystal-liquid phase transition presents key features such as (1) fast switching of crystal-liquid phases for both forward and backward reactions and (2) a high contrast ratio of viscoelasticity. In the characterization, POM is advantageous in offering information on the spatial distribution of LC molecule orientations, determining the type of liquid crystalline phases appearing in the material, and studying the orientation of LCs. Photorheometry allows measurement of a material's rheological properties under light stimuli and can reveal the photorheological switching properties of materials. Photo-DSC is a technique to investigate thermodynamic information of materials in darkness and under light irradiation. Lastly, XRD allows studying of microscopic structures of materials. The goal of this article is to clearly present how to prepare and measure the discussed properties of a photorheological material.

\section{Video Link}

The video component of this article can be found at https://www.jove.com/video/60433/

\section{Introduction}

Smart mechanical materials with the capability to change their viscoelastic properties in response to environmental variation have generated tremendous interest among researchers. Switchability is considered to be the most important material factor, which offers robustness of repetitive mechanical response in living organisms. To date, artificial switchable materials with versatile functions have been designed by utilizing soft matter (i.e., photoresponsive hydrogels ${ }^{1,2,3}$, polymers ${ }^{4,5,6,7,8,9,10,11}$, liquid crystals [LCs] ${ }^{9,10,11,12,13,14,15,16,17}$, pH-responsive micelles ${ }^{18,19,20,21,22}$ and surfactants ${ }^{23}$ ). However, these materials suffer from more than one of the following problems: lack of reversibility, low switching contrast ratio of viscoelasticity, low adaptivity, and slow switching speed. In conventional materials, a tradeoff exists between the switching contrast ratio of viscoelasticity and switching speed; thus, designing materials covering all of these criteria with high performance is challenging. To realize materials with the aforementioned omnicapability, selecting or designing molecules that carry emergent natures of both high fluidity (viscous property) and rigidity (elastic property) is essential.

Liquid crystals are ideal systems with a potentially large number of liquid crystalline and solid phases that can be tuned by molecular design. This allows for self-assembled structures at different length scales in particular LC phases. For example, while high-symmetry nematic LCs (NLCs) exhibit low viscosity and elasticity because of their short-range spatial order, low-symmetry columnar or smectic LCs show high viscosity and elasticity due to one- and two-dimensional long-range periodicities. It is expected that if LC materials can be switched between two phases with large differences in their viscoelastic properties, then a viscoelastic smart material with high performance can be achieved. A few examples have been reported , $10,11,12,13,14,15^{\text {. }}$

This article demonstrates the preparation of a photorheological LC material with a phase sequence of isotropic (I)-nematic (N)-twist-bend nematic (TB) ${ }^{24}$-crystal (Cry) upon cooling (and vice versa upon heating), which exhibits fast and reversible viscoelastic switching in response to 
light. Presented here are the methods for measuring viscoelasticity and an illustration of the microscopic structure-viscoelasticity relationship. Details are described in the representative results and discussion sections.

Protocol

\section{Preparation of rubbed surfaces for aligning LC molecules planarly}

1. Prepare clean glass substrates.

1. Cut glass substrates using a diamond-based glass cutter (Table of Materials) into small square pieces with averages sizes of $1 \mathrm{~cm} x$ $1 \mathrm{~cm}$. Wash them by sonication at $38 \mathrm{kHz}$ or $42 \mathrm{kHz}$ in an alkaline detergent (Table of Materials, diluted in water at a detergent:water volume ratio of 1:3) and rinse with distilled water repeatedly (typically, more than 10x with 5 min of sonication for each rinse).

2. Subject the substrates to ultraviolet-ozone $\left(\mathrm{UV}-\mathrm{O}_{3}\right)$ cleaner (Table of Materials) for more than $10 \mathrm{~min}$

2. Coat planar alignment layer onto clean glass substrates.

1. Drip $20 \mu \mathrm{L}$ of $1 \mathrm{~mL}$ of a polyimide planar alignment solution (Table of Materials, used as is) with a pipette onto the cleaned glass substrates. Immediately spin-coat the solution, using a spin coater (Table of Materials) at 3,000 rpm and room temperature (RT) for 70 s.

NOTE: The typical thickness of the alignment layer is about $20 \mathrm{~nm}$.

2. Bake the coated glass substrates at $80^{\circ} \mathrm{C}$ for $60 \mathrm{~min}$ to remove the solvent and at $180{ }^{\circ} \mathrm{C}$ for $>60$ min for curing. Rub the substrates using a rayon-cloth rubbing machine (Table of Materials) with the following parameters: rotation speed $=300 \mathrm{rpm}$, plate speed $=20$ $\mathrm{mm} / \mathrm{s}$, and impression $=0.3 \mathrm{~mm}$ to realize uniaxial alignment of LC materials.

\section{Preparation of LC cells}

1. Place a glass substrate coated with the alignment layer onto another substrate, with the alignment layers face-to-face, and ensure that they are $80 \%$ overlapped to form a cell.

NOTE: The $20 \%$ nonoverlapped surfaces are to be used for introducing LC materials into the cell.

2. Place $100 \mu \mathrm{L}$ of a photoreactive adhesive (Table of Materials) and $0.1 \mathrm{mg}$ of micrometer-sized glass particles (diameter $=5 \mu \mathrm{m}$ ) onto a clean glass substrate and mix them manually using the tip of a paper clip. Move the mixed material to four corners of the cell to adjust the cell gap and illuminate the cell using a low-pressure mercury vapor short arc lamp (Table of Materials) with a wavelength of $365 \mathrm{~nm}\left(1.1 \mathrm{~W} / \mathrm{cm}^{2}\right)$. Place the cell under the LED lamp at a distance of $1 \mathrm{~cm}$ for $5 \mathrm{~min}$.

3. After illumination, place the cell onto a hot stage and set the target temperature of the stage to heat the cell to a temperature above the isotropic liquid (I)-nematic $(\mathrm{N})$ phase transition (typically at $160^{\circ} \mathrm{C}$ ). Transfer the LC material (1-[4-butoxyazobenzene-4'-yloxy]-6-[4cyanobiphenyl-4' yl]hexane; CB6OABOBu; $0.2-10.0 \mu \mathrm{L}$ ) onto one open surface of the cell and push the materials towards the cell entrance using a microspatula to obtain contact between the LC material and entrance of the cell. Wait for the LC materials to be filled in the cell by capillary force.

NOTE: CB6OABOBu has a phase sequence: Cry $100.3^{\circ} \mathrm{C}$ TB $105.2^{\circ} \mathrm{C} \mathrm{N} 151.7^{\circ} \mathrm{C}$ I on heating and I $151.4^{\circ} \mathrm{C} \mathrm{N} 104.5^{\circ} \mathrm{C}$ TB $83{ }^{\circ} \mathrm{C}$ Cry on cooling. Do not introduce $\mathrm{CB} 6 \mathrm{OABOBu}$ into the $\mathrm{N}$ phase or TB phase because flow-induced alignment is promoted.

\section{Texture characterization by polarizing optical microscopy}

1. Observe the LC cells placed on the hot stage to control the sample temperature $\left(40-180^{\circ} \mathrm{C}\right)$ with $\pm 0.1 \mathrm{~K}$ accuracy under a polarizing light microscope (POM, Table of Materials) using 4x-100x objective lenses. Record textures using a digital color camera sequentially during cooling and heating.

2. Use a UV epi-illuminator (Table of Materials) equipped on the POM with a wavelength of $365 \mathrm{~nm}\left(50 \mathrm{~mW} / \mathrm{cm}^{2}\right)$.

\section{Photorheological measurements}

1. Prepare of rheological measurements.

1. Before placing any sample onto the stage of the rheometer (Table of Materials), perform geometry inertia calibration and zero gap calibration controlled by a software according to manufacturer's instructions to ensure accuracy of the rheological study. Weigh $250 \mathrm{mg}$ of the CB6OABOBu powder sample and load it onto the base quartz plate of the rheometer. NOTE: For the present study, a plate with a diameter of $50 \mathrm{~mm}$ is used.

2. Set the temperature of the sample chamber to a value above the I-N phase transition point $\left(>160^{\circ} \mathrm{C}\right)$. Set a gap value for approaching the measuring plate to the base quartz plate to sandwich the sample (typical gap value used $=20 \mu \mathrm{m}$ ). Trim excess sample (e.g., by using paper wipes) that is outside of the gap when the measuring plate stops at the trimming position, which is $25 \mu \mathrm{m}$ above the targeted gap.

NOTE: Do not allow excess amount of $\mathrm{CB} 6 \mathrm{OABOBu}$ to be introduced to the sample chamber, as this makes the measurements inaccurate.

2. Perform rheological measurements.

1. Irradiate UV light at $365 \mathrm{~nm}\left(1-100 \mathrm{~mW} / \mathrm{cm}^{2}\right)$, measuring photorheological switching of CB6OABOBu using the high-pressure mercury vapor short arc lamp.

NOTE: The light will be guided from beneath the sample container through the base quartz plate. 
2. Perform measurements in 1) oscillatory mode for extracting dynamic restoring information of the material and 2) steady rotational mode for obtaining effective rotational viscosity. For measurements in rotational mode, apply a constant shear stress of $13 \mathrm{~Pa}$ to the sample to ensure that the measurement is made in the Newtonian regime.

NOTE: The selection of the modes is performed by a software according to the manufacturer's instructions.

\section{Photo-differential scanning calorimetry}

1. Weigh $10 \mathrm{mg}$ of $\mathrm{CB} 60 \mathrm{ABOBu}$ powder sample and load it into a gold differential scanning calorimetry (DSC) pan. Heat the sample to $170{ }^{\circ} \mathrm{C}$ in the isotropic phase and ensure that there is no inhomogeneous sample distribution in the DSC pan as observed by the naked eye. Cover the DSC pan with a quartz plate.

2. Perform photo-DSC measurements according to the manufacturer's instructions (Table of Materials). Measure DSC data at a scan of $10{ }^{\circ} \mathrm{C} /$ $\min$.

NOTE: The photo-DSC machine is equipped with a UV light intensity of $50 \mathrm{~mW} / \mathrm{cm}^{2}$.

\section{X-ray diffraction characterization}

1. Heat the powder $\mathrm{CB} 6 \mathrm{OABOBu}$ sample using the hot stage at $170{ }^{\circ} \mathrm{C}$ and suck the sample into an $\mathrm{XRD}$ capillary (diameter $=0.5 \mathrm{~mm}$ ) by capillary force.

2. Attach the capillary to a sample holder equipped with a temperature controller. Set the chamber temperature $\left(60^{\circ} \mathrm{C}, 70^{\circ} \mathrm{C}, 80^{\circ} \mathrm{C}, 90^{\circ} \mathrm{C}, 100\right.$ ${ }^{\circ} \mathrm{C}, 110^{\circ} \mathrm{C}, 120^{\circ} \mathrm{C}, 130{ }^{\circ} \mathrm{C}, 140^{\circ} \mathrm{C}, 150^{\circ} \mathrm{C}, 160^{\circ} \mathrm{C}$, and $170{ }^{\circ} \mathrm{C}$ for each X-ray diffraction measurement).

3. Irradiate the sample by X-ray and detect the diffracted X-ray beams by a detector without UV irradiation and under a UV light intensity of 10 $\mathrm{mW} / \mathrm{cm}^{2}$ for $1 \mathrm{~min}$ and $10 \mathrm{~min}$.

NOTE: The current study was conducted in RIKEN beamline BL45XU. The light source was the SPring-8 standard in-vacuum undulator. A liquid nitrogen-cooled Si double crystal monochromator was used to monochromatize the beam. The wavelength was $1 \AA$.

\section{Representative Results}

POM images, photorheometric data, photo-DSC data, and XRD intensity profiles were collected in darkness during temperature variation and while shining UV light. Figure 1a,b represents the structure of $\mathrm{CB} 6 \mathrm{OABOBu}$, with its phase sequence and possible conformations optimized by the MM2 forcefield in the modeling program (e.g., ChemBio3D).

When $\mathrm{CB} 6 \mathrm{OABOBu}$ is in the trans-state, two energy-plausible conformational states appear, and the twisted conformation is the most stable one that promotes formation of the TB phase. When CB6OABOBu is excited to the cis-state when exposed to UV light, a kink-conformation is realized. Though the current conformational optimization made by the modeling program is useful for determining the conformation of a single molecule, it cannot be used for simulating conformational state of multiple molecules that are interacting, or even for self-assemblies of larger molecular clusters.

Figure 1c,d shows the POM textures in darkness and under $30 \mathrm{~mW} \mathrm{~cm} \mathrm{~cm}^{-2} \mathrm{UV}$ irradiance, during cooling of the sample in a $2 \mu \mathrm{m}$-thick LC cell with uniformly rubbed planar alignment. In the $\mathrm{N}$ phase, uniaxial alignment of molecules is realized (Figure 1c, top). When decreasing the temperature to the TB in darkness, a striped pattern forms, at which the stripes run parallel to the rubbing direction of the LC cell (Figure 1c, middle). This stripe pattern arises as a result of buckling instability and is recognized as a symbol of the TB phase, first reported by Panov et al. $^{25}$. Further decreasing of the temperature leads to crystallization (Figure 1c, bottom). Irradiation of the UV light alters conformation from the trans- to cis-state, resulting in phase variation and thus texture variation. If starting from the TB phase, the UV light transforms the striped texture to the uniaxially aligned state of the $\mathrm{N}$ phase (Figure 1d, top-middle). Turning off the UV light allows the molecules to relax and reenter the transstate, and the striped texture of the TB phase forms again.

Figure 2 shows the effective viscosity of $\mathrm{CB} 6 \mathrm{ABOBu}$ under various conditions measured by the rheometer. Figure $2 \mathrm{a}$ shows the temperature dependence of the effective shear viscosity. The reason for calling the measured viscosity the effective shear viscosity is that the real components of viscosity in liquid crystals are orientation-dependent, and the measured viscosity is an orientation-averaged value in the current study. Figure $\mathbf{2 b}$ shows the shear stress dependence of the effective shear viscosity at different temperatures during the first and second runs. Figure 2c presents variation among the effective shear viscosity triggered by UV irradiation at different temperatures. Figure $\mathbf{2 d}$ demonstrates switching curves of the effective shear viscosity in a log scale at two different temperatures, (i.e., one in the $\mathrm{N}$ phase and the other in the TB phase). The detailed temperature dependence of the switching times is summarized in Table 1.

Figure $\mathbf{3 a}, \mathbf{b}$ shows the textures of $\mathrm{CB} 6 \mathrm{OABOBu}$ in a nonaligned sample under $50 \mathrm{~mW} / \mathrm{cm}^{2} \mathrm{UV}$ irradiance at $80{ }^{\circ} \mathrm{C}($ Figure $3 \mathbf{a})$ and after cooling to $60{ }^{\circ} \mathrm{C}$ (Figure $3 \mathrm{~b}$ ). Photo-DSC curves of Figure $3 \mathrm{c}$ demonstrate that upon cooling, I-N phase transitions of the trans- and cis-isomers are different. Though the photo-DSC is useful for detecting differences between the dark- and light-stimulated states, it should be noted that photoDSC usually makes it difficult to quantitatively compare the real heat flow of the differences, since the baseline of the DSC curves changes significantly due to light absorption by the sample and the metal surface of the DSC pan. Figure 3d demonstrates that upon heating, the melting of the crystal phase of the trans- and cis-isomers are different, as measured by conventional DSC. Figure 3e,f shows XRD diffraction plots of the diffracted intensity as a function of d-spacing without and with UV irradiation, respectively. It can be seen that the intensity at each peak changes drastically when UV light is irradiated, mainly attributed to the crystalline structural transformation and local melting. 
a

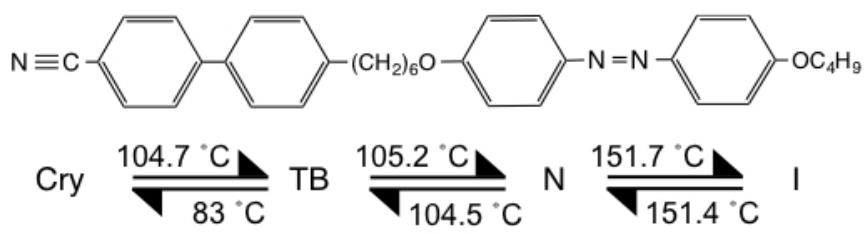

b

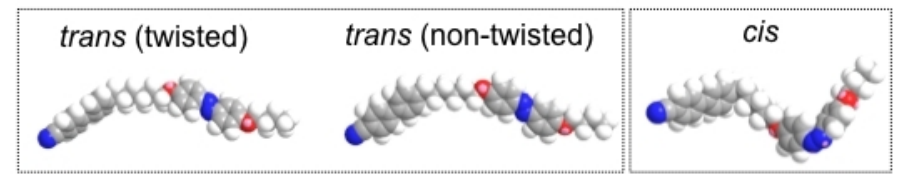

C
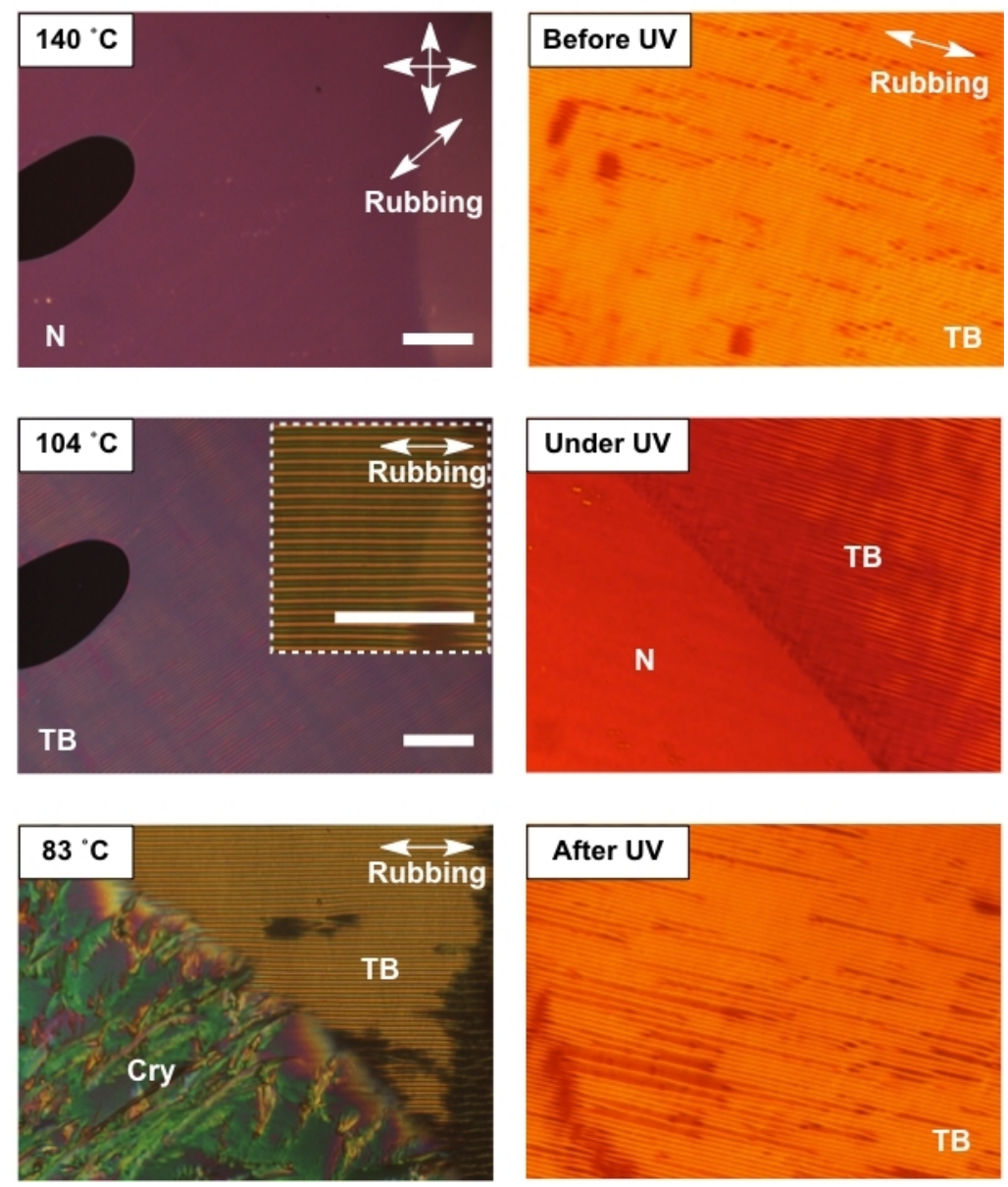

Figure 1: Chemical structure of $\mathrm{CB} 6 \mathrm{OABOBu}$ and the evolution of textures on cooling.(a) Chemical structure of $\mathrm{CB} 6 \mathrm{OABOBu}$ and its phase sequence. (b) Space-filling molecular models of CB6OABOBu optimized by the MM2 forcefield in the modeling program. (c) POM textures of $\mathrm{CB} 6 \mathrm{OABOBu}$ under crossed polarizers in a $2 \mu \mathrm{m}$-thick cell with uniformly rubbed planar alignment; during cooling without UV illumination. Top: in the $\mathrm{N}$ phase at $140{ }^{\circ} \mathrm{C}$; middle: in the TB phase at $104^{\circ} \mathrm{C}$; bottom: at the TB-Cry phase transition. (d) POM textures at $90{ }^{\circ} \mathrm{C}$ illustrating the photoswitching process. Top: before UV; middle: coexistence of $\mathrm{N}$ and TB phases shortly after $30 \mathrm{~mW} / \mathrm{cm}^{2} \mathrm{UV}$ irradiance at $365 \mathrm{~nm}$; bottom: relaxed TB texture after switching off the UV illumination. Scale bars represent $100 \mu \mathrm{m}$. This figure has been adapted with permission from Aya et al. ${ }^{26}$. Please click here to view a larger version of this figure. 
a

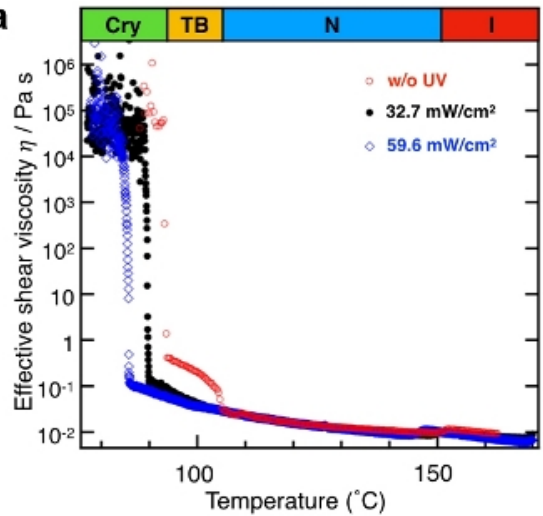

C

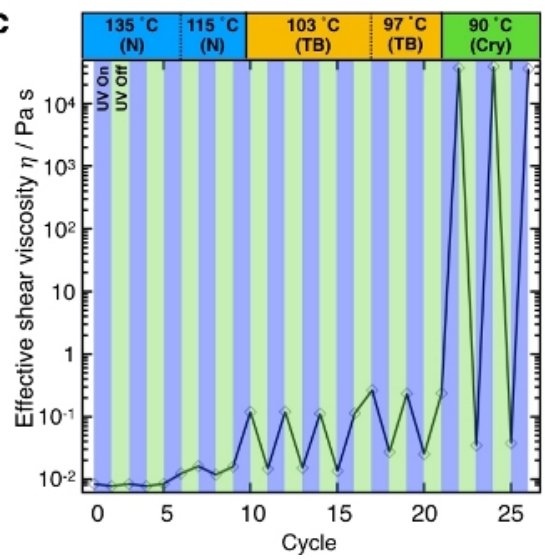

b

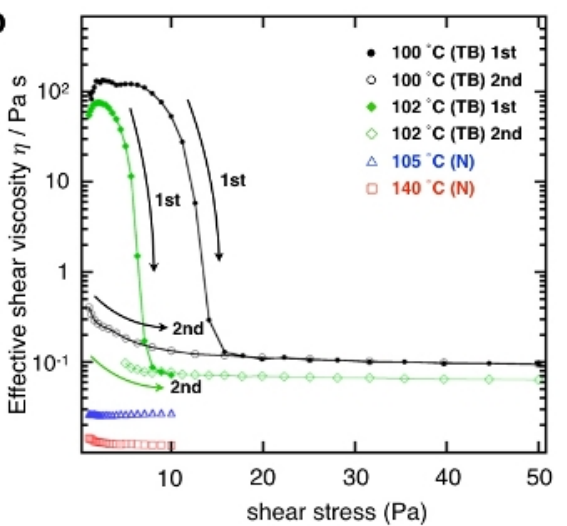

d

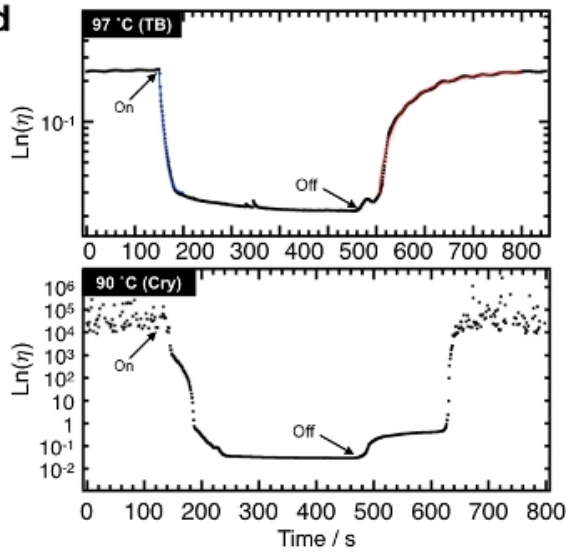

Figure 2: Rheological properties and photodynamics of photoswitching of the rheological properties of CB6OABOBu. (a) Temperature dependence of the effective shear viscosity measured at a constant shear stress of $13 \mathrm{~Pa}$ in rotational mode with different UV irradiances: 0 mW/ $\mathrm{cm}^{2}$ (red circles), $32.7 \mathrm{~mW} / \mathrm{cm}^{2}$ (black circles), and $59.6 \mathrm{~mW} / \mathrm{cm}^{2}$ (blue diamonds). (b) The effective shear viscosity as a function of increasing shear stress at selected temperatures. Black filled circles $\left(100^{\circ} \mathrm{C}\right)$ and green filled diamonds $\left(102{ }^{\circ} \mathrm{C}\right)$ are data measured on the first scan, while black open circles $\left(100^{\circ} \mathrm{C}\right)$ and green open diamonds $\left(102^{\circ} \mathrm{C}\right)$ are the data measured on the second scan. (c) Repeatable photoswitching of the effective shear viscosity at $59.6 \mathrm{~mW} / \mathrm{cm}^{2}$ irradiance. High and low values in each temperature correspond to UV-OFF and UV-ON states. (d) Photoswitching of the effective shear viscosity shown on a log scale at $97^{\circ} \mathrm{C}$ in the TB phase and $90{ }^{\circ} \mathrm{C}$ in the Cry phase. Blue and red solid lines for the TB phase are best-fitting curves using simple exponential function upon UV-ON and UV-OFF states. The UV intensity is $59.6 \mathrm{~mW} /$ $\mathrm{cm}^{2}$. This figure has been modified and adapted with permission from Aya et al. ${ }^{26}$. Please click here to view a larger version of this figure. 
a

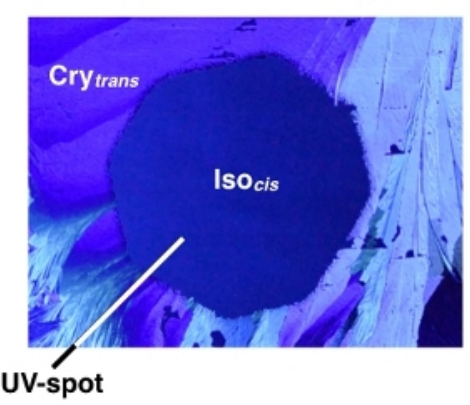

C

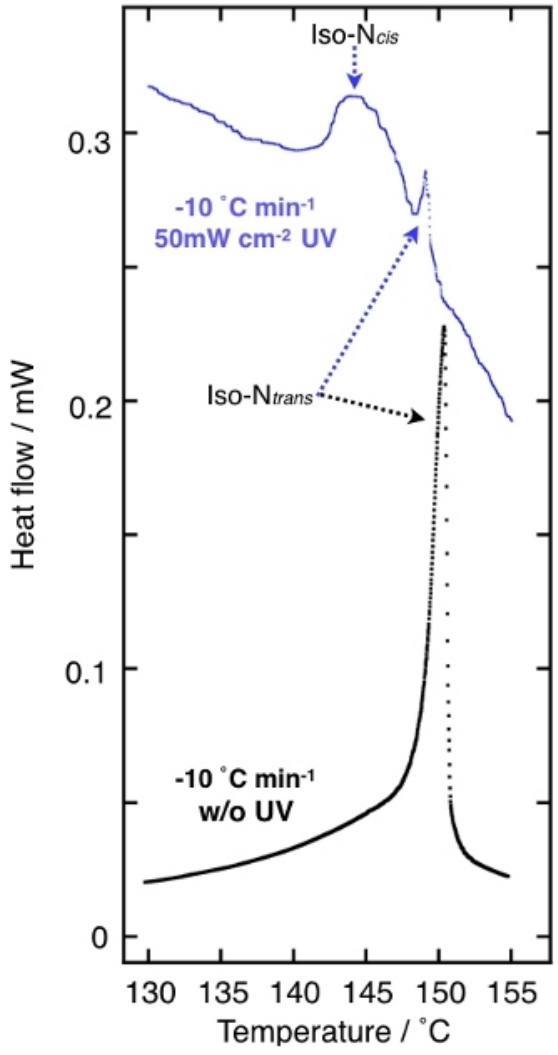

e

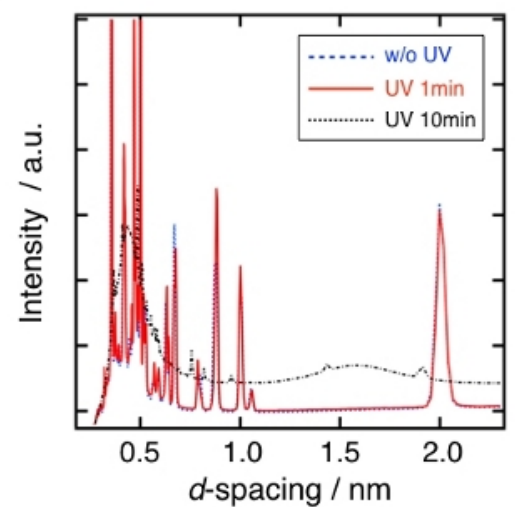

b

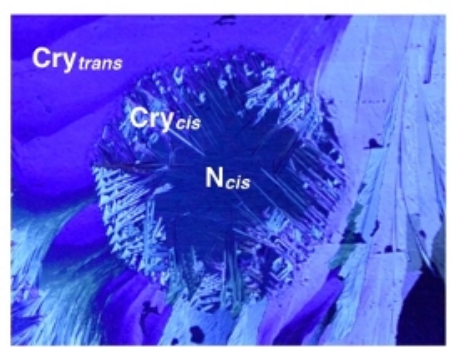

d

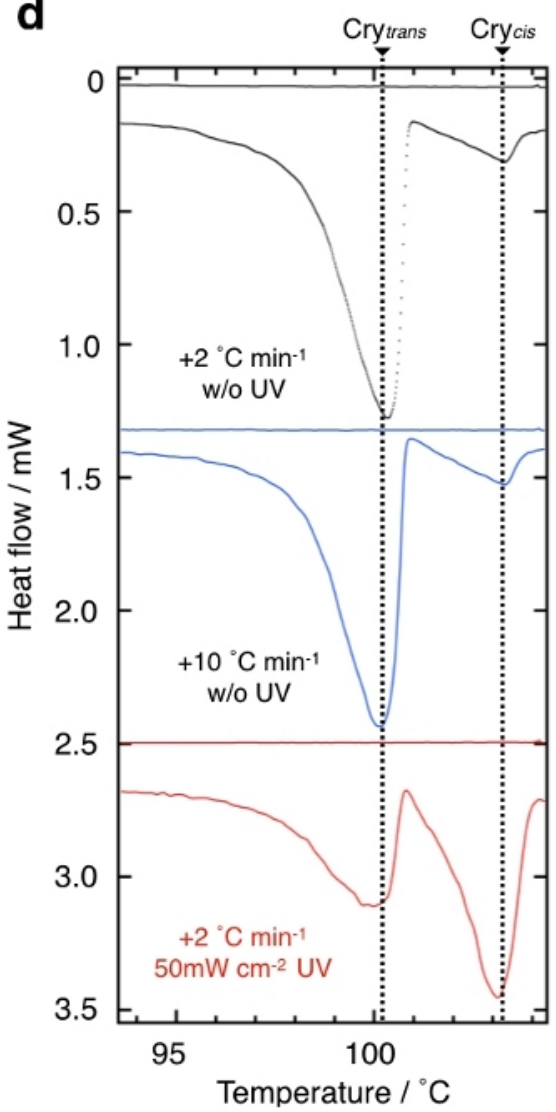

f

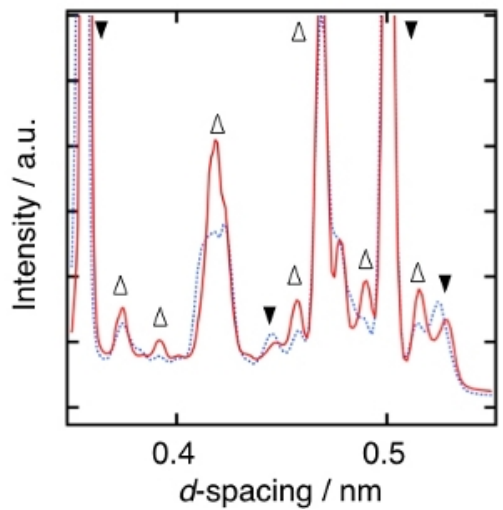

Figure 3: Evidence of existence of micro-segregated domains with different crystal structures in the Cry phase. (a,b) POM textures through blue filter under $50 \mathrm{~mW} / \mathrm{cm}^{2}$ UV irradiance in an octagonal spot of the shape of the field iris diaphragm in the middle at (a) $80{ }^{\circ} \mathrm{C}$ and (b) $60{ }^{\circ} \mathrm{C}$. (c) Temperature dependences of the heat flow of the sample during cooling at a rate of $10^{\circ} \mathrm{C} / \mathrm{min}$ without UV (black dots) and under UV (blue dots). (d) Temperature dependences of the heat flow of the trans-rich sample during heating at $2{ }^{\circ} \mathrm{C} / \mathrm{min}$ and $10^{\circ} \mathrm{C} / \mathrm{min}$ rates (black and blue curves, respectively) without UV, and of the cis-rich sample at $2{ }^{\circ} \mathrm{C} / \mathrm{min}$ rate (red curve). (e,f) Shown is d-spacing dependence of the wide 
angle X-ray diffraction intensity. (f) Magnified view of the small d-value region of panel e. Blue dashed, red solid, and black long-dashed lines indicate the X-ray diffraction profiles without UV illumination, under $10 \mathrm{~mW} / \mathrm{cm}^{2}$ irradiance for $1 \mathrm{~min}$ and 10 min, respectively. Open upward and filled downward triangles show increases and decreases in the diffracted intensity of each peak. This figure has been modified and adapted with permission from Aya et al. ${ }^{26}$. Please click here to view a larger version of this figure.

\section{Discussion}

As revealed in Figure 1, CB6OABOBu is a photo-responsive material with I, N, TB, and Cry phase sequences upon cooling. Since local ordering of these phases differs significantly, the photo-driven switching of rheological properties is expected to exhibit good viscoelastic contrast. To quantitatively investigate this, photo-rheology measurements were performed.

First, we consider the rheological data measured in the dark (Figure 2a, red open circles). At the I-N phase transition, the effective viscosity ( $\eta_{\text {eff }}$ ) decreases, which is attributed to a shear-induced flow alignment. In the $\mathrm{N}$ phase, the viscosity is practically independent of the shear stress, indicating a Newtonian fluid behavior (Figure $\mathbf{2 b}$ ). Transitioning to the TB phase results in an increase of the effective shear viscosity by one order of magnitude. Considering that the TB phase has a local nematic ordering but exhibits pseudo-layer structure analogous to the smectic ordering, the increase of the effective shear viscosity is attributed to formation of the pseudo-layer structures.

In the TB phase, strong shear thinning is observed with clear threshold values as a result of realignment of the pseudo-layer structures (Figure 2b). Subsequent solidification of the sample results in a sharp jump in $\eta_{\text {eff }}(\propto$ shear stress if shear rate is kept constant) by five orders of magnitude. The large scattering of the shear viscosity data in the crystal phase is a result of the sample's large resistance exerted on the rotating cone. The sample, in this regime, is a solid characterized by storage modulus instead of a fluid characterized by viscosity. Results under UV intensities of $32.7 \mathrm{~mW} / \mathrm{cm}^{2}$ and $59.6 \mathrm{~mW} / \mathrm{cm}^{2}$ are shown as black filled circles and blue open diamonds. Three main differences are observed between this data and that measured in darkness: 1) a downshift of transition temperatures, 2) a decrease in $\eta_{\text {eff }}$ in each phase, and 3) no significant viscosity variation among the original N-TB transition temperature for illuminated samples, which is explained by the disappearance of the TB phase under UV light.

It is clear that the rheological properties are indeed significantly distinct in different phases. To test the photo-driven rheological switching, rheological measurements were performed by shining UV light onto the sample. Figure 2c reveals that the photo-driven rheological switching has different contrast values at different temperatures: nearly 1 in the I and $\mathrm{N}$ phases, 10 in the TB phase, and $10^{6}$ in the Cry phase. The ON and OFF switching times are also very short ( 100 s, ON and OFF switching times shown in Table 1) both in the TB and Cry phases. The switching time is defined as the transient time for the variation of effective viscosity from $90 \%$ to $10 \%$ of its original value (that before the UV irradiation). Since the contrast in different phases is different, the switching time cannot be fairly compared between different phases. It is worth noting that for other molten liquids, the initial crystal phase typically recovers within several hours to several days, since their high viscosity prevents backward reaction in bulk, even at high temperatures ${ }^{9,14}$.

To determine the reason for the absence of slow nucleation, POM observation, photo-DSC, and XRD measurements were conducted. As the POM images in Figure 3 show, shining UV in the Cry phase triggers melting to the I phase at $80{ }^{\circ} \mathrm{C}$ (cis-state rich). Maintaining the UV irradiation while decreasing the temperature causes crystallization of the cis-state molecules to occur at different temperatures than those of the transstate. This suggests a microsegregation of trans- and cis-states. Photo-DSC data give direct evidence for this. As Figure 3c,d shows, exposure to UV light results in splitting of the phase transition peaks for both the I-N (on cooling) and crystal melting (on heating). These confirm that trans- and cis-state molecules form different phase structures. Thus far, most of the explored photo liquefactions owe their origins to the photoinduced temperature shift of glass transition. In contrast, this work demonstrates a novel working mechanism in realizing fast photo liquefaction processes, except for some recent discoveries ${ }^{27,28}$.

\section{Disclosures}

The authors have nothing to disclose.

\section{Acknowledgments}

This work was supported by the HAS-JSPS bilateral joint research project. Financial support from grants NKFIH PD 121019 and FK 125134 is acknowledged.

\section{References}

1. Grindy, S. C., Holten-Andersen, N. Bio-inspired metal-coordinate hydrogels with programmable viscoelastic material functions controlled by longwave UV light. Soft Matter. 13, 4057-4065 (2017).

2. Rosales, A. M., Mabry, K. M., Nehls, E. M., Anseth, K. S. Photoresponsive elastic properties of azobenzene-containing poly(ethylene-glycol)based hydrogels. Biomacromolecules. 16, 798-806 (2015).

3. Chang, D., Yan, W., Yang, Y., Wang, Q., Zou, L. Reversible light-controllable intelligent gel based on simple spiropyran-doped with biocompatible lecithin. Dyes and Pigments. 134, 186-189 (2015).

4. Irie, M., Hirano, Y., Hashimoto, S., Hayashi, K. Photoresponsive Polymers. 2. Reversible Solution Viscosity Change of Polymamides Having Azobenzene Residues in the Main Chain. Macromolecules. 14, 262-267 (1981).

5. Ito, S., Akiyama, H., Sekizawa, R., Mori, M., Yoshida, M., Kihara, H. Light-Induced Reworkable Adhesives Based on ABA-type Triblock Copolymers with Azopolymer Termini. ACS Applied Materials and Interfaces. 10, 32649-32658 (2018).

6. Yamamoto, T., Norikane, Y., Akiyama, H. Photochemical liquefaction and softening in molecular materials, polymers, and related compounds. Polymer Journal. 50, 551-562 (2018). 
7. Petr, M., Helgeson, M. E., Soulages, J., McKinley, G. H., Hammond, P. T. Rapid Viscoelastic Switching of an Ambient Temperature Range Photoresponsive Azobenzene Side-chain Liquid Crystal Polymer. Polymer. 54, 2850-2856 (2013).

8. Han, G. G. D., Li, H., Grossman, J. C. Optically controlled long-term storage and release of thermal energy in phase-change materials. Nature Communications. 8, 1446-1-10 (2017).

9. Akiyama, H., Yoshida, M. Photochemically Reversible Liquefaction and Solidification of Single Compounds Based on a Sugar Alcohol Scaffold with Multi Azo-Arms. Advanced Materials. 24, 2353-2356 (2012).

10. Akiyama, H. et al. Photochemically reversible liquefaction and solidification of multiazobenzene sugar-alcohol derivatives and application to reworkable adhesives. ACS Applied Materials and Interfaces. 6, 7933-7941 (2014).

11. Akiyama, H., Fukata, T., Yamashita, A., Yoshida, M., Kihara, H. Reworkable adhesives composed of photoresponsive azobenzene polymer for glass substrates. Journal of Adhesion. 93, 823-830 (2017).

12. Norikane, Y. et al. Photoinduced Crystal-to-Liquid Phase Transitions of Azobenzene Derivatives and Their Application in Photolithography Processes through a Solid-Liquid Patterning. Organic Letters. 16, 5012-5015 (2014).

13. Kim, D.Y., Lee, S.A., Kim, H., Kim, S. M., Kim, N., Jeong, K.U. An azobenzene-based photochromic liquid crystalline amphiphile for a remotecontrollable light shutter. Chemical Communications. 51, 11080 (2015)

14. Saito, S. et al. Light-melt adhesive based on dynamic carbon frameworks in a columnar liquid-crystal phase. Nature Communications. 7 , 12094-1-7 (2016).

15. Peng, S., Guo, Q., Hughes, T. C., Hartley, P. G. Reversible Photorheological Lyotropic Liquid Crystals. Langmuir. 30, 866-872 (2014)

16. Ito, S., Yamashita, A., Akiyama, H., Kihara, H., Yoshida, M. Azobenzene-Based (Meth)acrylates: Controlled Radical Polymerization, Photoresponsive Solid-Liquid Phase Transition Behavior, and Application to Reworkable Adhesives. Macromolecules. 51, 3243-3253 (2018)

17. Yue, Y., Norikane, Y., Azumi, R., Koyama, E. Light-induced mechanical response in crosslinked liquid-crystalline polymers with photoswitchable glass transition temperatures. Nature Communications. 9, 3234-1-8 (2018).

18. Lee, H.Y., Diehn, K. K., Sun, K., Chen, T., Raghavan, S. R. Reversible Photorheological Fluids Based on Spiropyran-Doped Reverse Micelles. Journal of the American Chemical Society. 133, 8461-8463 (2011).

19. Su, X., Cunningham, M. F., Jessop, P. G. Switchable viscosity triggered by $\mathrm{CO} 2$ using smart worm-like micelles. Chemical Communications. 49, 2655-2657 (2013)

20. Cho, M.Y., Kim, J.S., Choi, H. J., Choi, S.B., Kim, G.W. Ultraviolet light-responsive photorheological fluids: as a new class of smart fluids Smart Materials and Structures. 26, 054007-1-8 (2017).

21. Oh, $\mathrm{H}$. et al. A simple route to fluids with photo-switchable viscosities based on a reversible transition between vesicles and wormlike micelles. Soft Matter. 9, 5025-5033 (2013).

22. Akamatsu, M. et al. Photoinduced viscosity control of lecithin-based reverse wormlike micellar systems using azobenzene derivatives. RSC Advances. 8, 23742- 23747 (2018).

23. Song, B., Hu, Y., Zhao, J. A single-component photo-responsive fluid based on a gemini surfactant with an azobenzene spacer. Journal of Colloid and Interface Science. 333, 820-822 (2009).

24. Borshch, V. et al. Nematic twist-bend phase with nanoscale modulation of molecular orientation. Nature Communications. 4, 2635-2643 (2013).

25. Panov, V. P. et al. Spontaneous Periodic Deformations in Nonchiral Planar-Aligned Bimesogens with a Nematic-Nematic Transition and a Negative Elastic Constant. Physical Review Letters. 105, 167801-1-4 (2010).

26. Aya, S. et al. Fast-and-Giant Photorheological Effect in a Liquid Crystal Dimer. Advanced Materials Interfaces. 6, 1802032-1-7 (2019).

27. Ishiba, K. et al. Photoliquefiable ionic crystals: A phase crossover approach for photon energy storage materials with functional multiplicity. Angewandte Chemie International Edition. 54, 1532-1536 (2015).

28. Zhou, $\mathrm{H}$. et al. Photoswitching of glass transition temperatures of azobenzene-containing polymers induces reversible solid-to-liquid transitions. Nature Chemistry. 9, 145-151 (2017). 